ESAIM: M2AN

Vol. 40, No 2, 2006, pp. 225-237

DOI: $10.1051 / \mathrm{m} 2 \mathrm{an}: 2006012$
ESAIM: Mathematical Modelling and Numerical Analysis

www.edpsciences.org/m2an

\title{
NUMERICAL EVIDENCE OF NONUNIQUENESS IN THE EVOLUTION OF VORTEX SHEETS
}

\author{
Milton C. Lopes Filho ${ }^{1}$, John Lowengrub ${ }^{2}$, Helena J. Nussenzveig Lopes ${ }^{1}$ \\ AND YUXI ZHENG ${ }^{3}$
}

\begin{abstract}
We consider a special configuration of vorticity that consists of a pair of externally tangent circular vortex sheets, each having a circularly symmetric core of bounded vorticity concentric to the sheet, and each core precisely balancing the vorticity mass of the sheet. This configuration is a stationary weak solution of the 2D incompressible Euler equations. We propose to perform numerical experiments to verify that certain approximations of this flow configuration converge to a non-stationary weak solution. Preliminary simulations presented here suggest this is indeed the case. We establish a convergence theorem for the vortex blob method that applies to this problem. This theorem and the preliminary calculations we carried out support the existence of two distinct weak solutions with the same initial data.
\end{abstract}

Mathematics Subject Classification. 35Q35, 65M12 (Secondary), 76B03 (Primary), 76M23.

Received: January 21, 2005.

\section{INTRODUCTION}

The existence of weak solutions of the incompressible two-dimensional Euler equations has been established for initial vorticities $\omega_{0} \in \mathcal{B} \mathcal{M}_{c}\left(\mathbb{R}^{2}\right) \cap H_{\text {loc }}^{-1}\left(\mathbb{R}^{2}\right)$ provided that the negative (or the positive) part of $\omega_{0}$ belongs to $L^{1}\left(\mathbb{R}^{2}\right) \cap H_{\text {loc }}^{-1}\left(\mathbb{R}^{2}\right)$ (see $\left.[6,22,28,33]\right)$ and when the initial vorticity is the odd extension of single-signed vorticity on a half plane [20]. However, uniqueness of weak solutions is only known for initial vorticities in $L^{\infty}\left(\mathbb{R}^{2}\right)$ (see [36]) or very close to $L^{\infty}$ (see $[4,34,37]$ ). The uniqueness of weak solutions with more general initial velocity/vorticity distributions remains an open question. In 1993, V. Scheffer [27] constructed a weak solution in which the velocity belongs to $L^{2}\left((-T, T) \times \mathbb{R}^{2}\right)$ with compact support in space-time. Subsequently, a similar example was produced by A. Shnirelman in [30], with a more explicit account of the analytical mechanism involved. Scheffer's and Shnirelman's examples rest on an inverse energy cascade where the energy "bubbles

\footnotetext{
Keywords and phrases. Nonuniqueness, vortex sheets, vortex methods, Euler equations.

1 Departamento de Matematica, IMECC-UNICAMP, Caixa Postal 6065, Campinas, SP 13081-970, Brasil. 
up" from the infinitesimal scales. These examples suggest nonuniqueness of weak solutions (namely, the weak solution consisting of velocity identically equal to zero) if one allows for sufficiently irregular flows.

Promising candidates for such scenarios of nonuniqueness are flows involving vortex sheets. Vortex sheets are idealized models of narrow shear layers and are surfaces of discontinuity in which the tangential component of the velocity has a jump across the surface while the normal velocity is continuous. Thus, the fluid slips along the sheet while the sheet moves with the flow. Note that the vorticity due to a vortex sheet is in $\mathcal{B} \mathcal{M}_{c}\left(\mathbb{R}^{2}\right) \cap H_{\text {loc }}^{-1}\left(\mathbb{R}^{2}\right)$ so that single-signed vortex sheets are weak solutions of the incompressible $2 \mathrm{D}$ Euler equations. We use $\mathcal{B M}_{c}\left(\mathbb{R}^{2}\right)$ to denote the space of compactly supported bounded Radon measures in $\mathbb{R}^{2}$, endowed with the total variation norm. The reason that vortex sheets are such good candidates for providing potential examples of nonuniqueness is that they are highly unstable due to the Kelvin-Helmholtz instability. This instability reveals itself through the roll-up of the sheet (and analogous narrow shear layer) into large scale vortices. In fact, initial value problems with vortex sheet initial data are ill-posed in the sense of Hadamard in a wide class of Sobolev spaces, see $[3,10,16,35]$, although in certain analytic function spaces the problem is well-posed for short times $[2,9,31]$.

Additional motivation for considering nonunique vortex sheet evolution comes from considering the corresponding electron sheet problem for the Vlasov-Poisson equations. Electron sheet evolution for the 1D VlasovPoisson equations bears much resemblance to vortex sheet evolution for the incompressible 2D Euler equations. For this electron sheet problem, Majda et al. explicitly constructed two distinct electron sheet (weak) solutions with the same initial data [23].

Finally, even further motivation for considering nonunique vortex sheet evolution comes from the intriguing results of Pullin [26] and Phillips and Pullin [25]. In [25,26], these authors exploited the difference in behavior of the vortex sheet problem in analytic and non-analytic function spaces to obtain numerical evidence of multiple self-similar vortex sheet solutions. For example, Pullin in [26] considered the self-similar evolution of a single, initially flat single-signed vortex sheet with $x$-coordinate given by $x(\Gamma, 0)=\operatorname{sgn}(\Gamma)(|\Gamma| / 2 a)^{1 / p}$ where $\Gamma$ is the circulation between a point $(x(\Gamma, 0), y(\Gamma, 0))$ and $(x(0,0), y(0,0))$. Further, $a$ is a constant and $0<p<2$. When $p=1$, the initial interface $x(\Gamma, 0)=\Gamma / 2 a$ is an analytic function of $\Gamma$ and the resulting vortex sheet is a stationary solution of the Euler equations. For all other values of $p$, there are singularities at the origin and the solutions are non-stationary. Pullin simulated the self-similar evolution of these vortex sheets by recasting the equations in self-similar form and solving the resulting integro-differential equations using several different numerical approximation techniques. Letting $p \rightarrow 1^{+}$, Pullin [26] and Phillips and Pullin [25] obtained a nontrivial self-similar single-spiral solution (in addition to the stationary solution). For $0.9666<p<1$, Pullin [26] obtained three different self-similar solutions - two single spiral and one double spiral solutions. While Pullin's evidence is suggestive of nonuniqueness for the initial value problem, it remains to obtain such an example directly.

In [14], Krasny performed a computational study for the elliptically loaded wing, showcasing roll-up of the sheet at the tips. The elliptically loaded wing is a classical example of a steady solution for the Birkhoff-Rott equations, and hence Krasny's experiments seemed to suggest nonuniqueness of vortex sheet evolution. This problem was further examined in [21], where it was shown that the steadily translating elliptically loaded wing is not a weak solution of the incompressible 2D Euler equations.

In this paper, we present preliminary numerical evidence and a rigorous study of convergence for the vortex blob method, supporting a scenario for the existence of multiple weak solutions of the initial value problem branching out from initial data containing smooth, single-signed vortex sheets.

The use of vortex blob methods to study vortex sheets dates from the work of Chorin [5] and Krasny [13]. In this approach, the vortex sheet is represented by a sum of vortex blobs that are generated by convolving the singular Birkhoff-Rott kernel with a smoothing function. The smoothing function depends on a regularization parameter that effectively controls the size of the blobs and the stability of the algorithm. Detailed numerical studies have been performed (e.g. $[13,15])$ that, among other things, indicate convergence to a weak solution of the Euler equations for single-signed vortex sheets. Very recently, Baker and Beale [1] have designed a class of 
high-order vortex blob methods for interfacial flows and suggest that the regularization parameter should scale with the local spacing of interfacial markers.

The specific initial vorticity configuration we consider is a case of two externally tangent confined eddies. A confined eddy is a compactly supported distribution of vorticity, with zero total vorticity, which is circularly symmetric with respect to some point in the plane. We mention that the limiting situation of a sequence of confined eddies with vanishing supports and constant total mass is called a phantom vortex by DiPerna and Majda [7]. As was observed in [7], confined eddies are stationary weak solutions of the incompressible 2D Euler equations. The velocity field associated with a confined eddy vanishes in the exterior of the support of the eddy. Hence, confined eddies may be externally superimposed, giving rise to an interesting class of stationary weak solutions of the 2D Euler equations, a property exploited in Greengard and Thomann [12]. It was proved in [18] that for initial vorticities consisting of two or more confined eddies with disjoint supports, the solution of the 2D incompressible Navier-Stokes equations converges back to the superposition of confined eddies as the viscosity vanishes. We comment in passing that it is easy to extend the convergence proof to handle the case in which the approximate solutions are generated by the vortex blob approximation and to handle the case in which the approximate solutions are obtained by evolving a smoothed version of the initial data.

The two confined eddies we consider in this paper, $\omega_{0}^{1}$ and $\omega_{0}^{2}$, are centered at $x^{1}$ and $x^{2}$ respectively. Each eddy consists of a uniform measure supported on the circle $\left|x-x^{i}\right|=\left|x^{1}-x^{2}\right| / 2$ (vortex sheet), with unit total measure, plus a core of bounded vorticity of total measure -1 , internal to each respective circle (see the $T=0$ plot in Fig. 1). We call this initial configuration osculating confined eddies. This flow configuration is a stationary weak solution of the incompressible 2D Euler equations. To see this, we recall that weak limits of stationary weak solutions are again stationary weak solutions. This fact is an instance of concentrationcancellation, proved first by DiPerna and Majda, see Theorems 3 and 3.1 in [8], see also Chapter 4, Theorem 7 in [11] for a simpler proof. Considering the specific situation of the osculating confined eddies, we approximate by separating the two confined eddies by a distance $\varepsilon>0$ and mollify, keeping the eddies separated.

The main purpose of the current work is to propose numerical experiments, using the vortex blob approximation, that would show evidence of existence of an additional, unsteady weak solution, evolving from this initial configuration. The preliminary numerical results presented in this paper support this conclusion.

We examine the issue of convergence of the vortex blob approximation to a weak solution of the incompressible 2D Euler equations. For our specific set of initial data this is an open problem, not covered by the convergence result in [17], since the overall vorticity field does not have a distinguished sign (note that the vortex sheets themselves are single-signed). The difficulty is somewhat surprising, since the vorticity is an $L^{\infty}$ perturbation of a nonnegative measure and falls well within the class of vorticities for which other approximation schemes give rise to a weak solution, namely, regularizing the initial data and exactly solving the 2D Euler equations or using the vanishing viscosity approximation, see $[6,22,28]$. The crux of this difficulty is that oppositely signed point vortices may attract one another and collide, leading to a singularity in the blob equations as the blob size converges to zero. Since like-signed vortices do not collide, collisions may be ruled out if the positive and negative vortices remain well-separated. Under this separation assumption, we show the convergence of the vortex blob approximation to a weak solution of the Euler equations. Our numerical simulations verify this separation of positive and negative vortices. Our result is an extension of the theory developed by Schochet in $[29]$.

It is reasonable to ask why one should search for additional examples of nonuniqueness, given Scheffer and Shnirelman's examples. The key issue is physical meaning. Given that Scheffer and Shnirelman's examples clearly rely on energy being spontaneously produced out of nothing, they cannot be viscous limits, an obvious criterion for being physically realizable. On the other hand, our unsteady weak solution is a limit of a recognizable approximation scheme which bears some resemblance with a vanishing viscosity limit and it may very well be the correct vanishing viscosity limit for the solutions of the $2 \mathrm{D}$ Navier-Stokes system with the osculating phantom vortices as initial data. Moreover, the family of approximations generated by the numerical experiment can be recognized as a rolling-up vortex sheet, something well within the physical context that the theory of weak solutions was intended to model. In particular, the approximations carry a uniform estimate 
in the total mass of vorticity and a sign condition on the singular part, which places our example within the class of flows covered by Delort's existence result. In contrast, Scheffer and Shnirelman's examples carry only an $L^{2}\left((0, T) ; H^{-1}\right)$ estimate on vorticity. Finally, these limit flows appear to conserve kinetic energy exactly, since we do not observe exponential concentration of vorticity at the roll-up points; see [19] for sharp conditions under which a weak solution conserves kinetic energy.

This paper is divided into four sections. In Section 2, the theoretical basis of our work is presented. In particular, we consider the existence of a subsequence of the vortex blob approximation which converges to a weak solution of the 2D Euler equations. In Section 3, we present the discretization of the confined eddies and show that they fall within the class covered by our convergence theorem. In Section 4, we briefly discuss our numerical experiments, present our computational results and draw some conclusions.

\section{Convergence of the vortex Blob method}

In this section we state and prove a convergence result for vortex blob approximations that applies to the numerical experiment under consideration. Let $\omega_{0} \in \mathcal{B} \mathcal{M}_{c}\left(\mathbb{R}^{2}\right) \cap H_{\text {loc }}^{-1}\left(\mathbb{R}^{2}\right)$ be such that $\omega_{0}=\omega_{0}^{+}-\omega_{0}^{-}$, where $\omega_{0}^{+}$and $\omega_{0}^{-}$are nonnegative bounded measures with disjoint compact supports.

We introduce the vortex blob approximation. We restrict our discussion to the Krasny blob function:

$$
\phi(x)=\frac{1}{\pi\left(1+|x|^{2}\right)^{2}}, x \in \mathbb{R}^{2},
$$

although what follows applies to any divisible blob function, see $[1,7]$. Define $\phi_{\eta}(x)=(1 / \eta)^{2} \phi(x / \eta)$ and denote

$$
K_{\eta}(x)=\frac{x^{\perp}}{2 \pi\left(|x|^{2}+\eta^{2}\right)}=K_{0} * \phi_{\eta}
$$

where $x^{\perp}=\left(-x_{2}, x_{1}\right)$ and $K_{0}$ is $K_{\eta}$ evaluated at $\eta=0$.

We discretize the initial vorticity $\omega_{0}$ by considering approximate vorticities $\widetilde{\omega}_{0, h}^{+}$and $\widetilde{\omega}_{0, h}^{-}$such that:

(H1) $\widetilde{\omega}_{0, h}^{ \pm} \rightarrow \omega_{0}^{ \pm}$weak-* in $\mathcal{B M}$, and there exists a single compact set containing the supports of $\widetilde{\omega}_{0, h}^{ \pm}$;

(H2) $\widetilde{\omega}_{0, h}^{ \pm}=\sum_{i=1}^{N^{ \pm}} \xi_{i, h}^{ \pm} \delta\left(x-\alpha_{i, h}^{ \pm}\right)$, where $N^{ \pm}=N^{ \pm}(h)$ and $\xi_{i, h}^{ \pm}>0$;

(H3) $\min _{i=1, \ldots, N^{+}, j=1, \ldots, N^{-}}\left|\alpha_{i, h}^{+}-\alpha_{j, h}^{-}\right|$is bounded away from zero, uniformly in $h$;

(H4) $\sum_{j, k=1, j \neq k}^{N^{ \pm}} \xi_{j, h}^{ \pm} \xi_{k, h}^{ \pm} \log \left|\alpha_{j, h}^{ \pm}-\alpha_{k, h}^{ \pm}\right|$is bounded, uniformly in $h$.

If the supports of the negative and positive parts of vorticity are initially disjoint, we can set up the initial discretization by choosing a rectangle containing the support of vorticity where we introduce a square mesh with cells $Q^{i j}$ and centers $\alpha^{i j}$ in such a way that in each $Q^{i j}$ the vorticity has a distinguished sign. We can construct discretizations:

$$
\widetilde{\omega}_{0, h}^{ \pm}=\sum\left(\int_{Q^{i j}} \omega^{ \pm} \mathrm{d} x\right) \delta\left(x-\alpha^{i j}\right),
$$

where $h$ is the diameter of $Q^{i j}$ and $\omega^{ \pm}$are the positive and negative parts of $\omega$. It is an easy consequence of Lemma 3.2 in [29] that the sequences $\widetilde{\omega}_{0, h}^{+}$and $\widetilde{\omega}_{0, h}^{-}$obtained satisfy the hypotheses (H1)-(H4).

The vortex blob system is given as follows. Let $X_{i, h, \eta}^{ \pm}(t)$ be the positions of the vortex blobs at time $t$. Then,

$$
\left\{\begin{aligned}
\frac{\mathrm{d}}{\mathrm{d} t} X_{i, h, \eta}^{ \pm} & =\sum_{j=1}^{N^{+}} \xi_{j, h}^{+} K_{\eta}\left(X_{i, h, \eta}^{ \pm}-X_{j, h, \eta}^{+}\right)-\sum_{j=1}^{N^{-}} \xi_{j, h}^{-} K_{\eta}\left(X_{i, h, \eta}^{ \pm}-X_{j, h, \eta}^{-}\right) \\
X_{i, h, \eta}^{ \pm}(0) & =\alpha_{i, h}^{ \pm} .
\end{aligned}\right.
$$


For each $\eta>0$ and $h>0$ fixed, the flux for this system of ODE's is smooth and globally bounded, together with its derivatives. This implies that the solution $X_{i, h, \eta}^{ \pm}(t)$ is defined for all time $t \geq 0$ and that there are no vortex collisions in finite time. We introduce our approximate vorticities by:

$$
\omega_{h, \eta}(x, t)=\omega_{h, \eta}^{+}(x, t)-\omega_{h, \eta}^{-}(x, t)=\sum_{j=1}^{N^{+}} \xi_{j, h}^{+} \phi_{\eta}\left(x-X_{j, h, \eta}^{+}\right)-\sum_{j=1}^{N^{-}} \xi_{j, h}^{-} \phi_{\eta}\left(x-X_{j, h, \eta}^{-}\right) .
$$

The approximate velocities are then given by $u_{h}=u_{h}(x, t)=K_{0} * \omega_{h, \eta}$. We fix a function $\eta=\eta(h)$ such that $\eta \rightarrow 0$ as $h \rightarrow 0$ and eliminate the explicit subscript $\eta$ from what follows.

We consider two notions of weak solutions to the incompressible 2D Euler equations. The first refers to the weak vorticity formulation:

$$
\int_{t} \int_{x} \varphi_{t}(x, t) \omega(x, t) \mathrm{d} x \mathrm{~d} t+\frac{1}{2} \int_{t} \int_{x} \int_{y}(\nabla \varphi(x, t)-\nabla \varphi(y, t)) \cdot \frac{(x-y)^{\perp}}{2 \pi|x-y|^{2}} \omega(x, t) \omega(y, t) \mathrm{d} x \mathrm{~d} y \mathrm{~d} t=0,
$$

for any test function $\varphi \in C_{0}^{\infty}\left(\mathbb{R}^{2} \times(0, T)\right)$, see [6,28]. The second is the weak velocity formulation which is perhaps the more standard weak formulation:

$$
\int_{t} \int_{x} \varphi_{t}(x, t) \cdot u(x, t)+(u(x, t))^{t} D \varphi(x, t) u(x, t) \mathrm{d} x \mathrm{~d} t=0
$$

for any divergence-free test vector field $\varphi \in\left(C_{0}^{\infty}\left(\mathbb{R}^{2} \times(0, T)\right)\right)^{2}$, together with the condition that $u$ be divergencefree in the sense of distributions, see [7]. The nonlinear term $u^{t} D \varphi u$ is the Jacobian matrix of $\varphi$, interpreted as a quadratic form and evaluated at $u$.

With the notation introduced above and assuming (H1)-(H4), we are now ready to state and prove our main result.

Theorem 1. Let $T>0$. Assume that there exists a constant $d>0$, independent of $h$ or $t \in[0, T]$, such that

$$
\min _{i=1, \ldots, N^{+}, j=1, \ldots, N^{-}, t \in[0, T]}\left|X_{i, h}^{+}(t)-X_{j, h}^{-}(t)\right| \geq d>0 .
$$

Then, any weak-* limit in $\mathcal{B M}$ of the sequence $\omega_{h}$ as $h \rightarrow 0$ is a weak solution of the weak vorticity formulation of the Euler equations. Furthermore, if the approximate velocities $u_{h}$ are uniformly bounded in $L^{\infty}\left([0, T] ; L_{\text {loc }}^{2}\left(\mathbb{R}^{2}\right)\right)$ then any weak limit of the sequence of approximate velocities is a weak solution of the weak velocity formulation of the Euler equations.

Proof. Our proof relies heavily on the work done in [29]. Using Lemma 2.1 in [29], it is enough to show the nonconcentration condition:

$$
\lim _{r \rightarrow 0} \lim _{h \rightarrow 0} \sup _{0 \leq t \leq T} \sup _{x \in \mathbb{R}^{2}}\left(\sum_{\left\{j \mid X_{j, h}^{ \pm}(t) \in B(x, r)\right\}} \xi_{j, h}^{ \pm}\right)=0 .
$$

Define the function:

$$
\psi_{\eta}=\psi_{\eta}(r)=\frac{1}{2} \log \frac{1}{r^{2}+\eta^{2}}
$$

and consider:

$$
\begin{gathered}
H_{h}^{ \pm}=\sum_{j, k=1}^{N^{ \pm}} \xi_{j, h}^{ \pm} \xi_{k, h}^{ \pm} \psi_{\eta}\left(\left|X_{j, h}^{ \pm}-X_{k, h}^{ \pm}\right|\right), \\
W_{h}^{ \pm}=\sum_{j, k=1}^{N^{ \pm}} \xi_{j, h}^{ \pm} \xi_{k, h}^{ \pm}\left|X_{j, h}^{ \pm}-X_{k, h}^{ \pm}\right|^{2},
\end{gathered}
$$




$$
\begin{gathered}
H_{h}^{I}=\sum_{j=1}^{N^{+}} \sum_{k=1}^{N^{-}} \xi_{j, h}^{+} \xi_{k, h}^{-} \psi_{\eta}\left(\left|X_{j, h}^{+}-X_{k, h}^{-}\right|\right), \\
W_{h}^{I}=\sum_{j=1}^{N^{+}} \sum_{k=1}^{N^{-}} \xi_{j, h}^{+} \xi_{k, h}^{-}\left|X_{j, h}^{+}-X_{k, h}^{-}\right|^{2} .
\end{gathered}
$$

We also introduce $J_{h}=J_{h}^{+}+J_{h}^{-}-2 J_{h}^{I}$, with $J_{h}^{ \pm}=H_{h}^{ \pm}+W_{h}^{ \pm}$and $J_{h}^{I}=H_{h}^{I}+W_{h}^{I}$. It is an immediate extension of an observation by Schochet in [29] that $J_{h}$ is a first integral of the vortex blob system. This first integral will provide us with the estimate needed to show the nonconcentration condition provided that we can control the evolution of the terms corresponding to the interaction of positive and negative vortices, i.e. $J_{h}^{I}$.

Assume without loss of generality that $d<1$ and that $\eta(h)<\sqrt{1-d^{2}}$, for every $0<h \leq 1$. From the separation assumption (2), we have:

$$
H_{h}^{I} \leq \frac{1}{2} \log \frac{1}{d^{2}+\eta^{2}} \sum_{j=1}^{N^{+}} \sum_{k=1}^{N^{-}} \xi_{j, h}^{+} \xi_{k, h}^{-} \leq \log \frac{1}{d}\left\|\widetilde{\omega}_{0, h}^{+}\right\|_{\mathcal{B} \mathcal{M}}\left\|\widetilde{\omega}_{0, h}^{-}\right\|_{\mathcal{B} \mathcal{M}}
$$

Next we estimate $W_{h}^{I}$. First we rewrite $W_{h}^{I}$ as:

$$
W_{h}^{I}=\sum_{j=1}^{N^{+}} \xi_{j, h}^{+}\left|X_{j, h}^{+}\right|^{2} \sum_{k=1}^{N^{-}} \xi_{k, h}^{-}+\sum_{k=1}^{N^{-}} \xi_{k, h}^{-}\left|X_{k, h}^{-}\right|^{2} \sum_{j=1}^{N^{+}} \xi_{j, h}^{+}-2\left(\sum_{k=1}^{N^{-}} \xi_{k, h}^{-} X_{k, h}^{-}\right) \cdot\left(\sum_{j=1}^{N^{+}} \xi_{j, h}^{+} X_{j, h}^{+}\right)
$$

Let:

$$
y^{ \pm}=y^{ \pm}(t) \equiv\left(\sum_{j=1}^{N^{+}} \xi_{j, h}^{+}\left|X_{j, h}^{+}\right|^{2}\right)^{1 / 2} .
$$

We observe that these are the only quantities which need to be estimated in order to estimate $W_{h}^{I}$. Indeed, using the Cauchy-Schwarz inequality, we obtain:

$$
\left|\sum_{j=1}^{N^{ \pm}} \xi_{j, h}^{ \pm} X_{j, h}^{ \pm}\right| \leq\left\|\widetilde{\omega}_{0, h}^{ \pm}\right\|_{\mathcal{B} \mathcal{M}}^{1 / 2} y^{ \pm}
$$

We have, for $y^{+}$:

$$
\begin{aligned}
\frac{\mathrm{d}}{\mathrm{d} t}\left(y^{+}\right)^{2} & =\sum_{j=1}^{N^{+}}\left(\xi_{j, h}^{+} 2 X_{j, h}^{+}\right) \cdot\left(\sum_{\ell=1}^{N^{+}} \xi_{\ell, h}^{+} K_{\eta}\left(X_{j, h}^{+}-X_{\ell, h}^{+}\right)-\sum_{\ell=1}^{N^{-}} \xi_{\ell, h}^{-} K_{\eta}\left(X_{j, h}^{+}-X_{\ell, h}^{-}\right)\right) \\
& =-2 \sum_{j=1}^{N^{+}} \sum_{\ell=1}^{N^{-}} \xi_{j, h}^{+} \xi_{\ell, h}^{-} X_{j, h}^{+} \cdot K_{\eta}\left(X_{j, h}^{+}-X_{\ell, h}^{-}\right),
\end{aligned}
$$

due to the antisymmetry of the kernel $K$ and the fact that $K_{\eta}(z) \cdot z=0$,

$$
\leq \frac{1}{\pi d}\left\|\widetilde{\omega}_{0, h}^{-}\right\|_{\mathcal{B} \mathcal{M}} \sum_{j=1}^{N^{+}} \xi_{j, h}^{+}\left|X_{j, h}^{+}\right|
$$


due to the separation assumption 2 ,

Therefore, since $y^{+}(t)>0$,

$$
\leq \frac{1}{\pi d}\left\|\widetilde{\omega}_{0, h}^{-}\right\|_{\mathcal{B M}}\left\|\widetilde{\omega}_{0, h}^{+}\right\|_{\mathcal{B M}}^{1 / 2} y^{+}
$$

for all $0 \leq t \leq T$.

$$
y^{+}(t) \leq \frac{1}{2 \pi d}\left\|\widetilde{\omega}_{0, h}^{-}\right\|_{\mathcal{B M}}\left\|\widetilde{\omega}_{0, h}^{+}\right\|_{\mathcal{B M}}^{1 / 2} T+y^{+}(0)
$$

Analogously,

for all $0 \leq t \leq T$.

$$
y^{-}(t) \leq \frac{1}{2 \pi d}\left\|\widetilde{\omega}_{0, h}^{+}\right\|_{\mathcal{B} \mathcal{M}}\left\|\widetilde{\omega}_{0, h}^{-}\right\|_{\mathcal{B M}}^{1 / 2} T+y^{-}(0)
$$

Hence, $W_{h}^{I} \leq C$, where $C$ is independent of $t$. Furthermore, since $\widetilde{\omega}_{0, h}^{ \pm} \rightarrow \omega_{0}^{ \pm}$weak-* in $\mathcal{B M}$, and since the supports of $\widetilde{\omega}_{0, h}^{ \pm}$are contained in a single compact set (hypothesis (H1)), it follows that the bounds obtained for $H_{h}^{I}$ and $W_{h}^{I}$ are independent of $h$ as well. In addition, using hypotheses (H1), (H4) and the separation assumption (2) together with the fact that $J_{h}$ is a conserved quantity, it follows that $J_{h}$ is bounded independently of time and $h$. Therefore we see that $J_{h}^{+}+J_{h}^{-}=J_{h}+2 J_{h}^{I}$ is a non-negative quantity which is uniformly bounded for $t \in[0, T]$ and $h \in(0,1]$.

Let $0<r<d / 2$ and $x \in \mathbb{R}^{2}$. Observe that there are either positive or negative vortices in $B(x, r)$, never both, due to the separation assumption (2). Hence we have:

$$
\left(\sum_{\left\{j \mid X_{j, h}^{ \pm} \in B(x, r)\right\}} \xi_{j, h}^{ \pm}\right)^{2} \leq \frac{-2}{\log \left(4 r^{2}+\eta^{2}\right)}\left(J_{h}^{+}+J_{h}^{-}\right) \leq \frac{-C}{\log \left(4 r^{2}+\eta^{2}\right)}
$$

Note that, since we assumed that $\eta<\sqrt{1-d^{2}}$ it follows that $4 r^{2}+\eta^{2}<1$.

The constant $C$ above is positive and independent of $t, x$ and $h$. Since the $\lim _{r \rightarrow 0} \lim _{h \rightarrow 0}$ of the right-handside above is zero, we thus have verified the nonconcentration condition.

Remark. Theorem 1 is, strictly speaking, a new existence result. As an existence result, it may not seem very interesting because the hypothesis of separation is very restrictive and artificial. However, Theorem 1 as a convergence result for the vortex blob method is interesting because the separation assumption can be easily verified numerically. The new feature in the proof is the a priori estimate of the terms corresponding to interaction of vorticities with opposite sign in the first integral $J^{h}$.

\section{Discretization of the initial Data}

Consider an initial measure $\omega_{0}$ consisting of one-dimensional Diracs supported on a pair of externally tangent unit circles (vortex sheets). More precisely, let $\omega_{0}^{+}$be given by:

$$
\left\langle\omega_{0}^{+}, \phi\right\rangle=\frac{1}{2 \pi} \int_{|x-(-1,0)|=1} \phi \mathrm{d} s+\frac{1}{2 \pi} \int_{|x-(1,0)|=1} \phi \mathrm{d} s
$$

for any $\phi \in C_{0}\left(\mathbb{R}^{2}\right)$. Consider also

$$
\omega_{0}^{-}=\frac{2}{\pi|x-(-1,0)|} \chi_{\{1 / 4<|x-(-1,0)|<1 / 2\}}+\frac{2}{\pi|x-(1,0)|} \chi_{\{1 / 4<|x-(1,0)|<1 / 2\}} .
$$

Our first observation is that $\omega_{0}=\omega_{0}^{+}-\omega_{0}^{-}$is a stationary weak solution of the incompressible 2D Euler equations, by virtue of being a superposition of confined eddies, see [18]. 
We consider an initial discretization of this measure as follows. Fix $K$ an integer. We choose the blob size $\eta^{2}=1 /(2 \sqrt{10} K)$. Let $N=10 K^{2}$, so that the square of the blob size is $1 /(2 \sqrt{N})$, and introduce:

$$
\begin{aligned}
\widetilde{\omega}_{0}^{+, N}(x)= & \sum_{i=0}^{N-1}(1 / N) \delta\left(x-\left(\cos (2 \pi i / N)-1-\eta^{2} / 4, \sin (2 \pi i / N)\right)\right) \\
& +\sum_{i=0}^{N-1}(1 / N) \delta\left(x-\left(\cos (2 \pi i / N)+1+\eta^{2} / 4, \sin (2 \pi i / N)\right)\right) .
\end{aligned}
$$

This is the discretization of the two vortex sheets. Note that they are no longer tangent but are separated by the distance $\eta^{2} / 2$.

We discretize the core of bounded vorticity by arranging the point vortices in a roughly uniform grid. Since the domain is the annulus $1 / 4<|x|<1 / 2$, the cores have an aspect ratio of about 10 in polar coordinates. We begin by choosing $K$ points in $[1 / 4,1 / 2]: r_{i}=1 / 4+(i-1) /(4(K-1))$ for $i=1, \ldots, K$. Next, we choose $10 K$ points in $[0,2 \pi]$, by taking $\theta_{j}=2 \pi(j-1) /(10 K)$, with $j=1, \ldots, 10 K$. We define $\alpha^{i j}=\left(r_{i} \cos \left(\theta_{j}\right), r_{i} \sin \left(\theta_{j}\right)\right)$ and

$$
\begin{aligned}
\widetilde{\omega}_{0}^{-, K}(x)= & \sum_{i=1}^{K} \sum_{j=1}^{10 K} \frac{1}{10 K^{2}}\left[\delta\left(x-\alpha^{i j}-\left(-1-\eta^{2} / 4,0\right)\right)\right. \\
& \left.+\delta\left(x-\alpha^{i j}-\left(1+\eta^{2} / 4,0\right)\right)\right] .
\end{aligned}
$$

Naturally, $\widetilde{\omega}_{0}^{K}=\widetilde{\omega}_{0}^{+, 10 K^{2}}-\widetilde{\omega}_{0}^{-,}$. In total, there are $M=40 K^{2}$ vortices. See the $T=0$ graph in Figure 1 for a plot of the vortices.

Proposition 1. The sequence of approximations $\left\{\widetilde{\omega}_{0}^{K}\right\}$ described above satisfies (H1)-(H4).

Proof. The hypothesis (H2) and (H3) are obviously satisfied by construction. Let us examine (H1). Let $\phi$ be a test function in $C_{0}\left(\mathbb{R}^{2}\right)$ and let $\langle\cdot, \cdot\rangle$ denote the duality pairing between $\mathcal{B M}$ and $C_{0}$. Then:

$$
\left\langle\widetilde{\omega}_{0}^{-, K}, \phi\right\rangle=\frac{2}{\pi} \sum_{i=1}^{K} \sum_{j=1}^{10 K} \frac{\pi}{20 K^{2}}\left(\phi\left(\alpha^{i j}+\left(1+\eta^{2} / 4,0\right)\right)+\phi\left(\alpha^{i j}-\left(1-\eta^{2} / 4,0\right)\right)\right),
$$

which is a pair of Riemann sums in polar coordinates, with small translations, and thus, since $\phi$ is continuous, converges as $K \rightarrow \infty$ to:

$$
\frac{2}{\pi} \int_{1 / 4}^{1 / 2} \int_{0}^{2 \pi} \phi(r \cos \theta+1, r \sin \theta)+\phi(r \cos \theta-1, r \sin \theta) \mathrm{d} \theta \mathrm{d} r \equiv \int \omega_{0}^{-} \phi \mathrm{d} x .
$$

The case of $\widetilde{\omega}_{0}^{+, 10 K^{2}}$ can also be identified with a pair of translated Riemann sums, converging to the appropriate limit.

Hypothesis (H4) is more delicate. For the negative vortices, checking hypothesis (H4) involves a simple adaptation of the proof of Lemma 3.2 in [29] since the grid in the bounded cores is comparable to a square grid and the weights $\xi_{j, h}^{-}$are precisely the total mass of $\omega_{0}^{-}$in each grid cell. The fact that the $\alpha_{j, h}^{-}$are not the centers of the grid cells, but one of their corners, is easy to deal with.

We now verify (H4) for the positive vortices. Let $\mathcal{H}_{N}$ be the logarithmic double sum involving the positive vortices that we wish to estimate. Let the complex position $z_{j N} \equiv \cos 2 \pi j / N+i \sin 2 \pi j / N$ for $j=0, \ldots, N-1$. We decompose this discrete pseudoenergy into self-induction and interaction terms for the two vortex sheets 
and center the calculation at the origin to obtain:

$$
\mathcal{H}_{N}=2 \sum_{j, k=1, j \neq k}^{N} \frac{1}{N^{2}} \log \left|z_{j N}-z_{k N}\right|+2 \sum_{j, k=1}^{N} \frac{1}{N^{2}} \log \left|z_{k N}-\left(z_{j N}+2+\eta^{2} / 2\right)\right| .
$$

We first compute the self-induction term:

$$
\begin{array}{r}
\sum_{j, k=1, j \neq k}^{N} \frac{1}{N^{2}} \log \left|z_{j N}-z_{k N}\right|=\sum_{j=1}^{N} \log \left|\prod_{k=1, k \neq j}^{N}\left(z_{j N}-z_{k N}\right)\right|= \\
\sum_{j=1}^{N} \log \left|\lim _{z \rightarrow z_{j N}} \frac{z^{N}-1}{z-z_{j N}}\right|=\frac{1}{N^{2}} \sum_{j=1}^{N} \log \left|N z_{j N}^{N-1}\right|=\frac{\log N}{N},
\end{array}
$$

which converges to 0 , and is therefore bounded, as $N \rightarrow \infty$.

Finally we estimate the interaction term. First observe that:

$$
\sum_{j, k=1}^{N} \frac{1}{N^{2}} \log \left|z_{k N}-\left(z_{j N}+2+\eta^{2} / 2\right)\right| \leq \log \left|4+\eta^{2} / 2\right|
$$

On the other hand,

$$
\begin{aligned}
& \sum_{j, k=1}^{N} \frac{1}{N^{2}} \log \left|z_{k N}-\left(z_{j N}+2+\eta^{2} / 2\right)\right|=\frac{1}{N^{2}} \sum_{j=1}^{N} \log \left|\left(z_{j N}+2+\eta^{2} / 2\right)^{N}-1\right| \geq \\
& \frac{1}{N^{2}} \sum_{j=1}^{N} \log \left(\left|z_{j N}+2+\eta^{2} / 2\right|^{N}-1\right) \geq \frac{1}{N^{2}} \sum_{j=1}^{N} \log N \eta^{2} / 2=\log \left(N \eta^{2} / 2\right)^{1 / N}
\end{aligned}
$$

Hence, if $\eta=\eta(N)$ does not vanish too fast, for example, if it is bounded below by a negative exponential, the pseudoenergy is uniformly bounded as $N \rightarrow \infty$. Of course, this is true in our case $\eta^{2}=1 /(2 \sqrt{N})$.

Therefore, Proposition 1 shows that if the separation assumption is valid, then Theorem 1 holds for the approximate solution sequences obtained by solving the vortex blob system (1) with the special initial conditions given above.

\section{Computational Results and COnClusions}

In this section, we briefly describe the actual experiments performed. We discretize equations (1) in time using a standard fourth-order Runge-Kutta time discretization. The time step considered was $\Delta t=0.01$, which was small enough to ensure temporal accuracy.

We now consider the evolution of our confined eddies. In Figure 1, a time sequence of the dynamics using $K=32$ is shown. This corresponds to $40960=40 \times 32^{2}$ point vortices which is the maximum number we used. Only the point vortices are graphed; there is no interpolation. The results suggest the existence of a nonstationary solution with initial data $\omega_{0}$. The positive and negative vortices remain well-separated throughout the simulation suggesting that the separation assumption (2) is valid.

Since we are concerned with the convergence in the limit $K \rightarrow \infty$, we next compare our results using $K=32$ to $K=16$ (10240 vortices), see Figure 2. We note that increasing $K$ leads to larger deformations of the eddies 

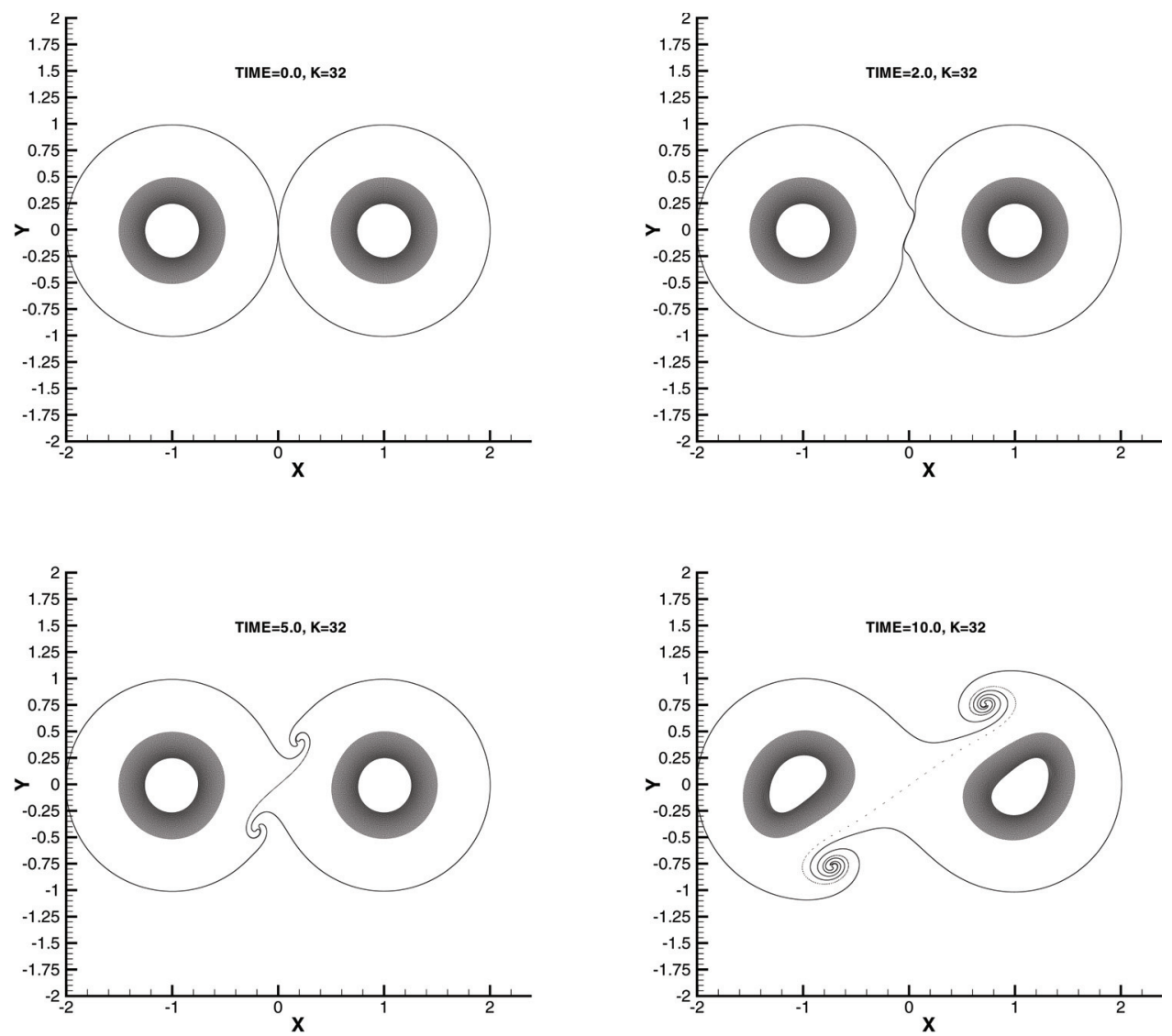

Figure 1. The evolution of the two confined eddies with $K=32$ which corresponds to $M=40960$ point vortices.

and hence enhancing non-stationarity. This behavior occurs consistently in comparison with lower resolutions as well.

The sequence of approximations presented here appears to be converging to a new nonstationary solution of the initial value problem. We can add quantitative information to this qualitative assessment by counting the number of vortices in a fixed disk centered at the origin. More precisely, we consider the integral of the vorticity $I_{\omega, K}(t)$ in a ball centered at the origin with radius 0.1 given by

$$
I_{\omega, K}(t)=\int_{|x|<.1} \widetilde{\omega}^{K}(x, t) \mathrm{d} x .
$$

This quantity is weakly lower semicontinuous with respect to weak-* convergence in Radon measures. Therefore, if $\lim \inf _{K} I_{\omega, K}(t)$ is smaller than its initial value, then any weak limit of the vorticity distribution is nonstationary. The quantity $I_{\omega, K}(t)$ is graphed in Figure 3 for several different resolutions. Observe that $I_{\omega, K}(t)$ is a decreasing function of both time and resolution $K$. The graphs strongly suggest convergence to a nonstationary vorticity distribution. A similar calculation, integrating against a smooth test function approximating the characteristic function of the ball of radius 1/10 used above would clearly yield the same results, while being continuous rather than semicontinuous.

These calculations were performed using a parallel implementation of the vortex blob method described here, run using 32 processors on a 256 processor IBM-SP2 at the University of Minnesota Supercomputer Institute. 

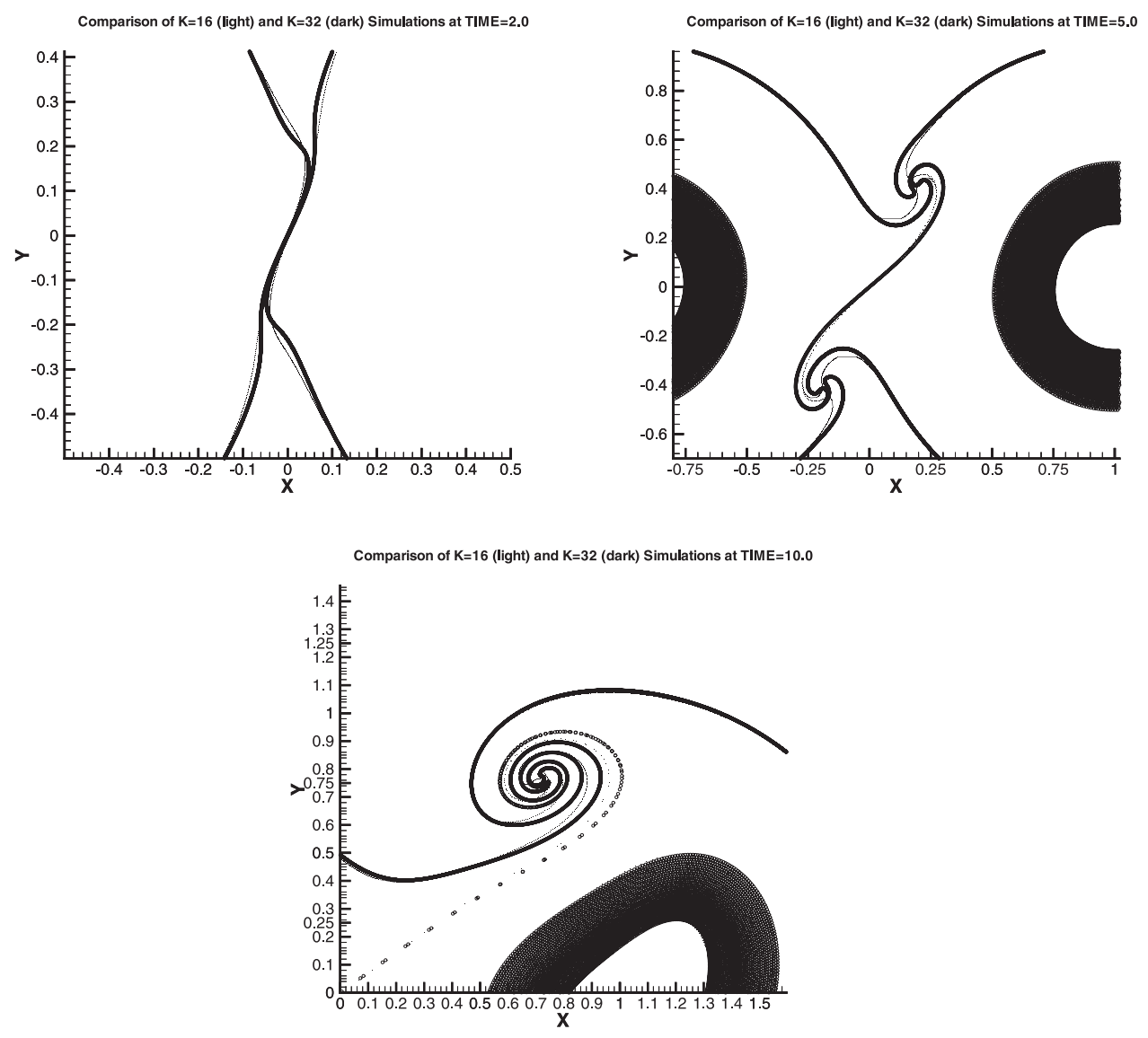

Figure 2. A comparison of the $K=16$ (light) and $K=32$ (dark) results in the regions of greatest deviation.

Our numerical evidence suggests that in the limit as $K \rightarrow \infty$, and hence $\eta \rightarrow 0$, the limiting vorticity distribution obtained from the vortex blob system is non-trivial and non-stationary. We regard the results obtained as a preliminary indication that the osculating confined eddies lead to nonuniqueness, but a more extensive numerical study is definitely warranted. The main purpose of this article is to suggest this example and to provide a rigorous theoretical framework for such experiments.

One may ask whether our computational solution may be obtained from the vanishing viscosity approximation. This is far from clear. While there have been studies comparing the inviscid roll-up of vortex sheets using the vortex blob approximation with simulations using small viscosity (see e.g. $[24,32]$ ), the connection between the blob and viscous regularizations is still not understood. The viscous regularization in the vortex sheet case does yield roll-up however. Thus, we conjecture that the vanishing viscosity approximation of the osculating confined eddies does yield a non-stationary weak solution of the 2D Euler equations.

Finally, regarding the numerical evidence presented herein, at least two possibilities should be considered. First, the convergence result proved was valid for infinite precision calculations, which leaves open the possibility that truncation error may be playing a role in our observations; second, the convergence result considers the limit of fine discretization, and the experiments performed here might not be resolved just yet. The experiment was designed with these objections in mind and appears to be converging as the discretization parameters are refined, but no amount of computational effort can dispel these possibilities entirely. In the absence of rigorous proof, additional heuristical evidence would certainly strengthen our case. For instance, we would propose as 


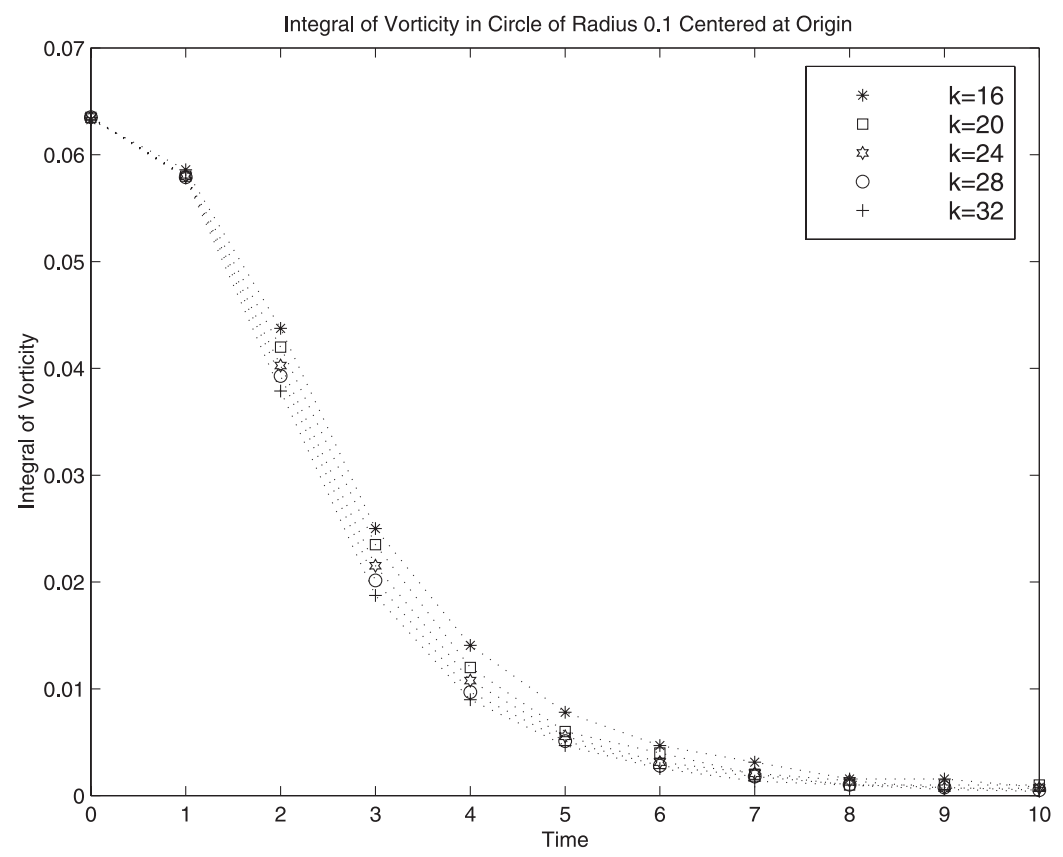

FiguRE 3. The integral of the vorticity in a circle of radius 0.1 centered at the origin. Several different resolutions $K$ are shown.

an interesting open problem to obtain a description the time-one map associated with a suitable regularization of the osculating confined eddies by means of asymptotic analysis.

Acknowledgements. The authors M.C. Lopes Filho and H.J. Nussenzveig Lopes thank the generous hospitality of the Dept. of Mathematics at Indiana University, where much of this research was done, and the I.M.A., for sponsoring several visits in connection with this work.

It is a pleasure to thank B. Cockburn for useful and stimulating discussions. We especially thank Qing Nie for his assistance in implementing the parallel algorithm to compute the vortex summations.

\section{REFERENCES}

[1] G.R. Baker and J.T. Beale, Vortex blob methods applied to interfacial motion. J. Comput. Phys. 196 (2004) $233-258$.

[2] R. Caflisch and O. Orellana, Long time existence for a slightly perturbed vortex sheet. Comm. Pure Appl. Math. 39 (1986) 807-838.

[3] R. Caflisch and O. Orellana, Singularity solutions and ill-posedness for the evolution of vortex sheets. SIAM J. Math. Anal. 20 (1989) 293-307.

[4] J.-Y. Chemin, A remark on the inviscid limit for two-dimensional incompressible fluids. Comm. Partial Differential Equations 21 (1996) 1771-1779.

[5] A. Chorin and P. Bernard, Discretization of a vortex sheet with an example of roll-up. J. Comput. Phys. 13 (1973) $423-429$.

[6] J.-M. Delort, Existence de nappes de tourbillon en dimension deux. J. Amer. Math. Soc. 4 (1991) 553-586.

[7] R. DiPerna and A. Majda, Concentrations and regularizations for 2-D incompressible flow. Comm. Pure Appl. Math. XL (1987) 301-345.

[8] R. DiPerna and A. Majda, Reduced Hausdorff dimension and concentration-cancellation for two-dimensional incompressible flow. J. Am. Math. Soc. 1 (1988) 59-95.

[9] J. Duchon and R. Robert, Global vortex sheet solutions of Euler equations in the plane. Comm. Partial Differential Equations 73 (1988) 215-224.

[10] D. Ebin, Ill-posedness of the Rayleigh-Taylor and Helmholtz problem for incompressible fluids. Comm. Partial Differential Equations 73 (1988) 1265-1295. 
[11] L.C. Evans, Weak convergence methods for nonlinear partial differential equations, CBMS Regional Conference Series in Mathematics 74 A.M.S., Providence, RI (1990).

[12] C. Greengard and E. Thomann, On DiPerna-Majda concentration sets for two-dimensional incompressible flow. Comm. Pure Appl. Math. 41 (1988) 295-303.

[13] R. Krasny, Desingularization of periodic vortex sheet roll-up. J. Comput. Phys. 65 (1986) 292-313.

[14] R. Krasny, Computation of vortex sheet roll-up in the Trefftz plane. J. Fluid Mech. 184 (1987) 123-155.

[15] R. Krasny and M. Nitsche, The onset of chaos in vortex sheet flow. J. Fluid Mech. 454 (2002) 47-69.

[16] G. Lebeau, Régularité du problème de Kelvin-Helmholtz pour l'équation d'Euler 2D. ESAIM: COCV 8 (2002) 801-825.

[17] J.G. Liu and Z.P. Xin, Convergence of vortex methods for weak solutions to the 2D Euler equations with vortex sheet data. Comm. Pure Appl. Math. XLVIII (1995) 611-628.

[18] M.C. Lopes Filho, H.J. Nussenzveig Lopes and Y.X. Zheng, Convergence of the vanishing viscosity approximation for superpositions of confined eddies. Commun. Math. Phys. 201 (1999) 291-304.

[19] M.C. Lopes Filho, H.J. Nussenzveig Lopes and E. Tadmor, Approximate solutions of the incompressible Euler equations with no concentrations. Ann. I. H. Poincaré-An. 17 (2000) 371-412.

[20] M.C. Lopes Filho, H.J. Nussenzveig Lopes and Z.P. Xin, Existence of vortex sheets with reflection symmetry in two space dimensions. Arch. Rational Mech. Anal. 158 (2001) 235-257.

[21] M.C. Lopes Filho, H.J. Nussenzveig Lopes and M.O. Souza, On the equation satisfied by a steady Prandtl-Munk vortex sheet. Comm. Math. Sci. 1 (2003) 68-73.

[22] A. Majda, Remarks on weak solutions for vortex sheets with a distinguished sign. Indiana U. Math J. 42 (1993) 921-939.

[23] A. Majda, G. Majda and Y.X. Zheng, Concentrations in the one-dimensional Vlasov-Poisson equations. I. Temporal development and non-unique weak solutions in the single component case. Physica D 74 (1994) 268-300.

[24] M. Nitsche, M.A. Taylor and R. Krasny, Comparison of regularizations of vortex sheet motion, Proc. 2nd MIT Conf. Comput. Fluid and Solid Mech., K.J. Bathe Ed., Elsevier, Cambridge, MA (2003).

[25] W.R.C. Phillips and D.I. Pullin, On a generalization of Kaden's problem. J. Fluid Mech. 104 (1981) 45-53.

[26] D.I. Pullin, On similarity flows containing two branched vortex sheets, in Mathematical Aspects of Vortex Dynamics, R. Caflisch Ed., SIAM (1989) 97-106.

[27] V. Scheffer, An inviscid flow with compact support in space-time. J. Geom. Anal. 3 (1993) 343-401.

[28] S. Schochet, The weak vorticity formulation of the 2D Euler equations and concentration-cancellation. Comm. P.D.E. 20 (1995) 1077-1104.

[29] S. Schochet, Point-vortex method for periodic weak solutions of the 2-D Euler equations. Comm. Pure Appl. Math. XLIX (1996) 911-965.

[30] A. Shnirelman, On the non-uniqueness of weak solutions of the Euler equations. Comm. Pure Appl. Math. L (1997) 1261-1286.

[31] P.L. Sulem, C. Sulem, C. Bardos and U. Frisch, Finite time analyticity for the two and three dimensional Kelvin-Helmholtz instability. Comm. Math. Phys. 80 (1981) 485-516.

[32] G. Tryggvason, W. Dahn and K. Sbeih, Fine structure of rollup by viscous and inviscid simulation. J. Fluids Eng.-T ASME 113 (1991) 31-36.

[33] I. Vecchi and S.J. Wu, On L ${ }^{1}$-vorticity for 2-D incompressible flow. Manuscripta Math. 78 (1993) 403-412.

[34] M. Vishik, Incompressible flows of an ideal fluid with vorticity in borderline spaces of Besov type. Ann. Sci. École Norm. S. Ser. 432 (1999) 769-812.

[35] S.J. Wu, Recent progress in mathematical analysis of vortex sheets, in Proceedings of the ICM, Beijing (2002) Vol. III, $233-242$.

[36] V. Yudovich, Non-stationary flow of an ideal incompressible liquid (in Russian), Zh. Vych. Mat. 3 (1963) 1032-1066.

[37] V. Yudovich, Uniqueness theorem for the basic nonstationary problem in the dynamics of an ideal, incompressible fluid. Math. Res. Lett. 2 (1995) 27-38. 